\title{
Detección de levaduras en cloaca de dos especies psitácidas nativas en un centro de rehabilitación en Chile
}

\author{
Detection of yeasts in cloacae of two species of native psittacine birds in \\ a Chilean rehabilitation center \\ G González-Heina, J González ${ }^{\text {b }}$ MC Díaz ${ }^{*}$ \\ aPrograma Doctorado en Ciencias Silvoagropecuarias y Veterinarias, Universidad de Chile, Santiago, Chile.

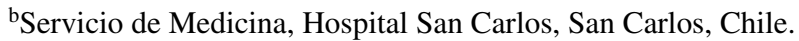 \\ 'Programa de Microbiología y Micología, ICBM, Facultad de Medicina, Universidad de Chile, Santiago, Chile.
}

\begin{abstract}
SUMMARY
The aim of this study was to evaluate the presence of Candida and Cryptococcus in Chilean psittacine birds. Twenty eight cloacae swab samples were collected from 131 captive psittacine birds kept in a rehabilitation wildlife center in Chile. The sampled birds were 24 Slender-billed parakeets (Enicognathus leptorhynchus) and 4 Austral parakeets (Enicognathus ferrugineus). Yeasts were recovered from 20 (71.4\%) of these 28 native psittacine birds. The most frequent yeasts isolated included Candida famata (8/28 samples), followed by Candida tropicalis (7/28), Cryptococcus albidus (3/28), Cryptococcus laurentii (2/28), Rhodotorula sp. (2/28), Candida glabrata (1/28) and Cryptococcus neoformans (1/28). The present study is the first report of yeasts in the E. ferrugineus and the endemic conure E. leptorhynchus.
\end{abstract}

Palabras clave: Candida sp., Cryptococcus sp, levaduras, psitácidas.

Key words: Candida sp., Cryptococcus sp, yeast, psittacine birds.

\section{INTRODUCCIÓN}

En Chile habitan naturalmente cuatro especies de psitácidas: Cyanoliseus patagonus (tricahue), Enicognathus ferrugineus (cachaña), Bolborhynchus aurifrons (periquito cordillerano) y la especie endémica Enicognathus leptorhynchus (choroy).

La microbiota del tracto digestivo de las aves está compuesta por bacterias, hongos, protozoos y por un determinado número de patógenos potenciales que influyen en el metabolismo y salud del hospedero (Bangert y col 1988). Las aves domésticas y silvestres pueden actuar como portadores asintomáticos de Cryptococcus y de otras levaduras también potencialmente patógenas para humanos como Candida y Rhodotorula sp. (Cafarchia y col 2006). Criptococosis y candidiasis constituyen, en aves, las principales infecciones oportunistas por levaduras, pero también se han descrito casos de enfermedad primaria en ellas (Velasco 2000). En la literatura nacional y extranjera revisada no existen reportes sobre la microbiota levaduriforme de cloaca de aves del género Enicognathus, pero sí existe un estudio bacteriano y fúngico de la cloaca de C. patagonus (Herrera 2001).

Aceptado: 18.11.2009.

* Código Postal 8389100, Clasificador 7, Universidad de Chile, Santiago, Chile; mcdiaz@med.uchile.cl
Cryptococcus neoformans tiene una distribución geográfica mundial, se encuentra comúnmente en las deyecciones aviares, muy raramente ocasiona problemas en el hospedero aviar y afecta en mayor grado a pacientes inmunocomprometidos (Doneley 1994). Otros Cryptococcus reportados en deyecciones de aves incluyen a $C$. albidus y $C$. laurentii, entre otras especies (Bangert y col 1988, Mattsson y col 1999, Cafarchia y col 2006, Cafarchia y col 2008, Rosario y col 2008).

C. neoformans y $C$. gattii en algunos casos se han asociado a la presencia de masas proliferativas que generan disrupción de las narinas, ramfoteca y estructuras del seno infraorbital en psitácidas (Doneley 1994, Raidal y Butler 2001, Malik y col 2003). Infecciones diseminadas por Cryptococcus también pueden afectar a diversas especies de psitácidas (Clipsham y Brit 1983, Rosskopf y col 1984, Fenwick y col 1985, Raso y col 2004).

Diversas especies del género Candida, incluidas Candida albicans, C. famata, C. glabrata entre otras, se han aislado de deyecciones aviares (Bangert y col 1988, Mancianti y col 2002). Por otro lado, C. tropicalis se ha logrado recuperar a partir de hisopados de cloaca de aves migratorias (Cafarchia y col 2006, Cafarchia y col 2008).

Candida es un agente oportunista en aves, pero puede llegar a actuar como un patógeno primario o secundario (Stacey 2006). Varias especies de Candida han sido recuperadas de lesiones en aves clínicamente afectadas (Panigraphy y col 1979, Keimer 1982, Velasco y col 2000, 
Kano y col 2001, Kunkle 2003, Osorio y col 2007). En aves, $C$. albicans se considera un invasor secundario que afecta principalmente la mucosa oral, esófago y buche (Oglesbee y col 1996). Las candidiasis también pueden afectar a proventrículo, estómago muscular e intestino delgado (Campbell 1986, Hubbard y col 1985). Lesiones atribuidas a Candida también han sido comunicadas en el tracto respiratorio, glándula uropigeal, pico y ojo de las aves (Tsai y col 1992, Oglesbee 1997). Candidiasis dérmicas y oculares se han comunicado en psitácidas (Tsai y col 1992, Carrasco y col 1993).

El objetivo del presente trabajo fue detectar la presencia de Candida y Cryptococcus en la cloaca de psitácidas nativas del género Enicognathus aparentemente sanas, de un centro de rehabilitación de fauna silvestre.

\section{MATERIAL Y MÉTODO}

\section{RECOLECCIÓN DE MUESTRAS}

En diciembre del 2007, de un total de 131 ejemplares de dos especies de psitácidas nativas mantenidas en cautiverio en siete jaulas no contiguas, en un centro de rehabilitación de fauna silvestre, se colectaron $28(21,4 \%)$ hisopados cloacales, pertenecientes a 4/17 E. ferrugineus y 24/114 E. leptorhynchus aparentemente sanas. El examen clínico evaluó condición corporal general, estado de mucosas, narinas y cloaca, resultando todas aparentemente sanas. $\mathrm{La}$ toma de muestra de cloaca se realizó con tórulas estériles previamente humedecidas en solución fisiológica estéril, luego fueron conservadas en suero fisiológico y procesadas antes de 24 horas de tomada la muestra.

\section{PROCESAMIENTO DE LAS MUESTRAS}

A partir del suero fisiológico, se sembró en placas de Agar Sabouraud glucosado a $37^{\circ} \mathrm{C}$ por 96 horas y en agar Staib más bifenilo $0,1 \%$ y se incubaron a $25^{\circ} \mathrm{C}$ por 14 días. Las colonias sospechosas se subcultivaron en Agar Sabouraud glucosado y agar Staib con bifenilo al $0,1 \%$ para obtener cultivos puros. La identificación de las colonias aisladas se basó en características macro y micromorfológicas, bioquímicas y fisiológicas. Las levaduras se identificaron como Cryptococcus sp., mediante la visualización microscópica de la cápsula con tinta china y pruebas bioquímicas (prueba de la ureasa, asimilación de hidratos de carbono y de nitrógeno) (Díaz y col 2007). La identificación de Candida sp. se realizó mediante la visualización micróscopica con lactofenol, prueba de tubo germinal, microcultivo y Auxonograma (Barnett y col 2000, Díaz y col 2007). En algunos casos se utilizaron otras pruebas bioquímicas: CHROmagar Candida, y galerías comerciales API: Api ® Candida, Biomérieux, Francia).

\section{RESULTADOS Y DISCUSIÓN}

De 28 hisopados de cloaca, se aislaron 24 (85,7\%) levaduras correspondientes a distintas especies del género Candida, Cryptococcus o Rhodotorula. Dentro del género Candida fue más frecuentemente aislada C. famata (8/28 muestras) seguida por $C$. tropicalis (7/28) y C. glabrata (1/28). Las especies de Cryptococcus correspondieron a $C$. albidus (3/28), C. laurentii (2/28) y $C$. neoformans (1/28) y Rhodotorula sp. se aisló en 2 ejemplares. De 7

Cuadro 1. Distribución de 24 levaduras aisladas de 28 muestras obtenidas de la cloaca de dos especies de psitácidas nativas. Santiago, Chile, 2007.

Distribution of yeast, isolated from 28 cloacae samples of two native psittacine species, Santiago, Chile, 2007.

\begin{tabular}{|c|c|c|c|c|}
\hline $\begin{array}{l}\text { Hongo } \\
\text { levaduriforme }\end{array}$ & Choroy & Cachaña & $\begin{array}{c}\text { Total levaduras } \\
\mathrm{N}^{\circ} \%\end{array}$ & Jaulas \\
\hline Candida famata & 7 & 1 & $8(28,57 \%)$ & $5 / 7$ \\
\hline Candida tropicalis & 5 & 2 & $7(25 \%)$ & $3 / 7$ \\
\hline Candida glabrata & 1 & 0 & $1(3,6 \%)$ & $1 / 7$ \\
\hline Total Candida & 13 & 3 & $16(57,14 \%)$ & $6 / 7$ \\
\hline Cryptococcus laurentii & 2 & 0 & $2(7,1 \%)$ & $2 / 7$ \\
\hline Cryptococcus albidus & 3 & 0 & $3(10,7 \%)$ & $3 / 7$ \\
\hline Cryptococcus neoformans & 1 & 0 & $1(3,57 \%)$ & $1 / 7$ \\
\hline Total Cryptococcus & 6 & 0 & $6(21,43 \%)$ & $5 / 7$ \\
\hline Rhodotorula sp. & 2 & 0 & $2(7,14 \%)$ & $2 / 7$ \\
\hline Total levaduras/total aves & $21 / 24$ & $3 / 4$ & $24 / 28(85,71)$ & $7 / 7$ \\
\hline
\end{tabular}


jaulas, Candida se detectó en aves provenientes de 6 jaulas, Cryptococcus de 5 y Rhodotorula de 2 jaulas.

De acuerdo al conocimiento de los autores el presente trabajo representa el primer estudio sobre detección de levaduras en la cloaca de E. ferrugineus y E. leptorhynchus. Las levaduras aisladas de cachañas y loros choroy fueron las mismas que se detectaron en un estudio realizado en cloaca de tricahues mantenidos en cautiverio: C. famata, C. albidus y Rhodotorula sp. (Herrera 2001), pero además en el presente estudio se encontraron otras especies como C. tropicalis y $C$. glabrata, que también han sido aisladas a partir de la cloaca y deyecciones de aves en cautiverio y migratorias (Mancianti y col 2002, Cafarchia y col 2006, Cafarchia y col 2008).

El aislamiento de levaduras $(85,7 \%)$ en el presente estudio es mayor al obtenido en una investigación realizada en 325 deyecciones procedentes de distintas especies de psitácidas en cautiverio (Italia), $(49,2 \%)$, en la que se detectó una mayor diversidad de especies dentro del género Candida (16 especies) y no se aisló Cryptococcus (Mancianti y col 2002). La gran variedad de especies de Candida aisladas podría atribuirse a que en este último estudio se examinaron deyecciones de una gran variedad de psitácidas pertenecientes a diversas colecciones privadas, además las deyecciones tuvieron contacto con el ambiente. El hecho de obtener cultivos negativos para Cryptococcus en medio Staib puede explicarse por no existir las condiciones ambientales en los aviarios para la sobrevida del microorganismo.

En el presente estudio, $57,1 \%$ de las levaduras aisladas a partir de 28 hisopados cloacales correspondieron a especies del género Candida y en 6 de los 7 aviarios al menos un ave fue positiva. No se detectó $C$. albicans, y tampoco $C$. parapsilosis, especies frecuentemente implicadas en candidiasis aviares (Oglesbee 1997, Velasco 2000, Kano y col 2001), pero sí C. tropicalis, la que también podría llegar a tener un rol patógeno en aves (Velasco 2000).

Se detectó C. neoformans en 3,6\% (1/28) de las muestras, similar a lo informado por Staib y Schulz-Dieterich (1984) en muestras de deyecciones desecadas procedentes de diferentes aves $(2,8 \%)$, lo que sugiere de acuerdo a la literatura que éstas pueden ser diseminadoras de esta levadura que constituye un potencial patógeno para el hombre (Staib y Schulz-Dieterich 1984). Infecciones por Cryptococcus en pacientes después de la exposición con aves han sido comunicadas (Littman y Borok 1968, Fessel 1993, Nosanchuk y col 2000, Bauters y col 2001, Shrestha y col 2004, Lagrou y col 2005).

En el presente estudio, dentro de los Cryptococcus fue más frecuentemente aislado $C$. albidus, levadura también detectada en la cloaca de $C$. patagonus (Herrera 2001). C. laurentii, microorganismo también aislado a partir de excrementos de otras especies de psitácidas (Bangert y col 1988, Mancianti y col 2002), fue recuperado a partir de dos E. leptorhynchus. En humanos, ambas levaduras también han sido descritas como potenciales patógenos (Kordossis y col 1998, Jover y col 2006).

El género Rhodotorula está ampliamente distribuido, y se encuentra en el ambiente, piel y en las mucosas normales del hombre y algunos animales (Torres-Rodríguez 1993). Las aves del presente estudio podrían llegar a actuar también como portadores de esta levadura oportunista, considerada parte de la microbiota transiente del tracto gastrointestinal aviar (Mancianti y col 2002). En el hospedero aviar, Rhodotorula también puede llegar a tener un rol patógeno, así $R$. mucilaginosa y $R$. glutinis han sido implicadas como el agente etiológico de dermatitis (Beemer y col 1970, Page y col 1976, Aruo 1980, Rosario y col 2008).

El presente estudio contribuye al conocimiento de la microbiota levaduriforme de Enicognathus sp., informando del aislamiento de una o más levaduras en el 71,4\% de los casos. En 15 de 28 Enicognathus sp. se detectaron especies del género Candida; en 1 E. leptorhynchus, C. neoformans y en 5 E. leptorhynchus, C. albidus y C. laurentii. Es factible que el alto porcentaje de aves positivas encontradas obedezca al sesgo de la muestra, a la metodología de aislamiento de la levadura y/o a las características de la población de Enicognathus sp. en estudio. Al estar éstas en un proceso de rehabilitación, pueden presentar un mayor grado de inmunodepresión que puede justificar este porcentaje de agentes oportunistas. Interesante sería ampliar el tamaño de muestra para realizar un estudio de prevalencia de Candida y Cryptococcus en cada una estas dos especies en cautiverio, como también el evaluar si existen diferencias significativas en la distribución de frecuencias de estas levaduras según especie aviar. Por otro lado, la evaluación de la microbiota levaduriforme de Enicognathus sp. silvestre libre para ser comparada con la de aves nativas psitácidas cautivas en zoológicos o centros de rehabilitación, podría aclarar el rol que juega el cautiverio.

Los resultados obtenidos de este estudio preliminar manifiestan también la necesidad de estudiar los factores de patogenicidad de los aislados (secreción fosfolipasa, síntesis de melanina, mayor grosor cápsula de polisacáridos, etc.) y el posible rol diseminador de levaduras al ambiente de las aves.

\section{RESUMEN}

Con el propósito de evaluar la presencia de Candida y Cryptococcus en una población de 131 psitácidas nativas mantenidas en cautiverio en un centro de rehabilitación de fauna silvestre localizado en Santiago, se colectaron 28 hisopados cloacales: 24 de choroy (Enicognathus leptorhynchus), especie endémica de Chile, y 4 de cachaña (Enicognathus ferrugineus). Veinticuatro levaduras se detectaron en 20 de los 28 Enicognathus sp. (71,4\%). Candida famata fue la más frecuentemente aislada (8/28 muestras), seguida por Candida tropicalis $(7 / 28)$, Cryptococcus laurentii (2/28), C. albidus (3/28), Rhodotorula sp. (2/28), Candida glabrata (1/28) y Cryptococcus neoformans (1/28). El presente estudio contribuye al conocimiento de la microbiota levaduriforme en aves psitácidas chilenas del género Enicognathus sp. 


\section{REFERENCIAS}

Aruo SK. 1980. Necrotizing cutaneous Rhodotorulosis in chickens in Uganda. Avian Dis 24, 1038-1043.

Bangert R, B Cho, P Widders, E Stauber, C Ward. 1988. A survey of aerobic bacteria and fungi in the feces of healthy psittacine birds. Avian Dis 32, 46-52.

Barnett JA, RW Payne, D Yarrow. 2000. Yeast: Characteristics and Identification, $3^{\text {rd }}$ ed. Cambridge University Press, Cambridge, UK.

Bauters TGM, M Moerman, G Pini, H Vermeersch, H Nelis. 2001. Colonization of a voice prosthesis by Cryptococcus neoformans. Med Mycol 39, 379-381.

Beemer A, S Schneerson-Porat, E Kuttin. 1970. Rhodotorula mucilaginosa dermatitis on feathered parts of chickens: an epizootic on poultry farm. Avian Dis 14, 234-239.

Cafarchia C, A Camarda, D Romito, M Campolo, N Quaglia, D Tullio, D Otranto. 2006. Ocurrence of yeasts in cloacae of migratory birds. Micopathologia 161, 229-234.

Cafarchia C, D Romito, C Coccioli, A Camarda, D Otranto. 2008. Phospholipase activity of yeasts from wild birds and possible implications of human disease. Med Mycol 46, 429-34.

Campbell TW. 1986. Mycotic diseases. In: Harrison GJ, Harrison LR (eds). Clinical Avian Medicine and Surgery. WB Saunders, Philadelphia, USA, Pp 464-472.

Carrasco L, MBautista, J De Las Mulas, H Jensen. 1993. Application of enzymeimmunohistochemistry for the diagnosis of Aspergillosis, Candidiasis, and Zygomycosis in three lovebirds. Avian Dis 37, 923-927.

Clipsham R, J Brit. 1983. Diseminated cryptococcosis in a green winged macaw (Ara). J Am Vet Med Assoc 183, 1303-1304.

Díaz MC, V Silva, G Hermosilla. 2007. Manual Práctico Curso Internacional de Micología Médica. Chile.

Doneley R. 1994. Cutaneous cryptococcus in an African grey parrot. Proc Assoc Avian Vet Aust Comm, Corrumbin, QLD, Pp 247.

Fenwick B, K Takeshita, A Wong. 1985. A moluccan cockatoo with disseminated cryptococcosis. J Am Vet Med Assoc 187, 1210-1212.

Fessel W. 1993. Cryptococcal meningitis after unusual exposures to birds. N Engl J Med 328, 1354-1355.

Herrera PG. 2001. Estudio microbiano y parasitario de coana y cloaca de loro tricahue (Cyanoliseus patagonus byroni) en cautiverio. Memoria de título, Escuela de Medicina Veterinaria, Universidad Santo Tomás, Chile.

Hubbard G, R Schmidt, D Eisenbrandt, WM Witt, K Fletcher. 1985. Fungal infections of ventriculi in captive birds. $J$ Wildl Dis 21, 25-28.

Jover A, C Ferrer, A Morán, J Gutiérrez, MF Colom. 2006. Criptococosis por Cryptococcus albidus en un paciente inmunocompetente. Rev Iberoam Micol 23, S3.

Kano R, Y Sakamoto, A Hanahachi, H Kamata, Y Fukuda, K Fujiwara, Hasegawa A. 2001. Molecular identification of C. parapsilosis from crop mucosa in a cockatiel. J Vet Diagn Invest 13, 437-439.

Keimer IF. 1982. Mycoses. In: Petrak LM (ed). Diseases of the cage and aviary. Lea and Febiger, Philadelphia, PA, USA, Pp 601-602.

Kordossis T, A Avlami, A Velegraki, I Stefanou, G Georgakopoulos, C Papalambrou, NJ Legakis. 1998. First report of Cryptococcus laurentii meningitis and a fatal case of Cryptococcus albidus cryptococcaemia in AIDS patients. Med Mycol 36, 335-339.

Kunkle R. 2003. Fungal infections. In: Saif YM, Barnes HJ, Glisson JR, Fadly AM, Mc Dougald LR, Swayne DE (eds). Diseases of poultry. $11^{\text {th }}$ ed. Blackwell Publishing Professional Ames IA, Iowa, USA, Pp 883-902.

Lagrou K, J Van Eldere, S Keuleers, F Hagen, R Merckx, J Verhaegen, WE Peetermans,T Boekhout. 2005. Zoonotic transmission of Cryptococcus neoformans from a magpie to an immunocompetent patient. J Intern Med 257, 385-388.
Littman M, R Borok. 1968. Relation of the pigeon to cryptococcosi: natural carrier state, heat resistance and survival of Cryptococcus neoformans. Mycopathologia 35, 329-345.

Malik R, M Krockenberger, G Cross, R Doneley, D Madill, D Black, P McWhirter, A Rozenwax, K Rose, M Alley, D Forshaw, I RussellBrown, A Johnstone, P Martin, C O'Brien, D Love. 2003. Avian Cryptococcosis. Med Mycol 41, 115-124.

Mancianti F, S Nardoni, R Ceccherelli. 2002. Occurrence of yeasts in psittacines droppings from captive birds in Italy. Mycopathologia $153,121-124$

Mattsson R, PD Haemig, B Olsen. 1999. Feral pigeons as carriers of Cryptococcus laurentii, Cryptococcus uniguttulatus and Debaryomyces hansenii. Med Mycol 37, 367-369.

Nosanchuk JD, S Shoham, B Fries, D Shapiro, S Levitz, A Casadevall. 2000. Evidence of zoonotic transmission of Cryptococcus neoformans from a pet cockatoo to an immunocompromised patient. Ann Intern Med 132, 205-208.

Oglesbee BL, S Mc Donald, K Warthen. 1996. Trastornos del sistema digestivo de las aves. In: Birchard S, Sherding R (eds). Manual Clínico de Pequeñas Especies. McGraw-Hill Interamericana, México, Pp 1541-1552.

Oglesbee BL. 1997. Mycotic Diseases. In: Altman R, Clubb S, Dorrestein G, Quesenberry K (eds). Avian Medicine and Surgery. Saunders, Philadelphia, USA, Pp 323-332.

Osorio C, O Fletcher, M Dykstra, K Post, J Barnes. 2007. Comb Candidiasis affecting roosters in a broiler breeder flock. Avian Dis $51,618-622$.

Page RK, O Fletcher, C Eidson, G Michaels. 1976. Dermatitis produced by Rhodotorula glutins in broiler-age chickens. Avian Dis 20, 416-421.

Panigraphy B, S Naqui, L Grumbles, C Hall. 1979. Candidiasis in cockatiel nestlings and mucormycosis in a pigeon. Avian Dis 23, 757-758.

Raidal S, R Butler. 2001. Chronic rhinosinusitis and rhamphotecal destruction in a Major Mitchell's Cockatoo (Cacatua leadbeateri) due to Cryptococcus neoformans var gattii. J Avian Med Surg 15, 121-125.

Raso T, K Werther, E Miranda, MJS Mendes-Giannini. 2004. Cryptococcosis outbreak in psittacine birds in Brazil. Med Mycol 42, 355-362.

Rosario I, B Acosta, F Colom. 2008. La paloma y otras aves como reservorio de Cryptococcus sp. Rev Iberoam Micol 25, S13-S18.

Rosskopf W, R Woerpel.1984. Cryptococcosis in a thick billed parrot. Proc Assoc Avian Vet, 281-288.

Shrestha RK, J Stoller, G Honari, G Procop, S Gordon. 2004. Pneumonia due to Cryptococcus neoformans in a patient receiving Infliximab: Possible zoonotic transmission from a pet cockatiel. Respiratory care 49, 606-608.

Stacey G. 2006. Evaluating and treating the gastrointestinal system. In: Harrison G, Lightfoot T (eds). Clinical Avian Medicine Vol 1. Spix Publishing, Inc., Palm Beach, Florida, USA, Pp 411-440.

Staib F, J. Schulz-Dieterich. 1984. Cryptococcus neoformans in fecal matter of birds kept in cages-control of Cryptococcus neoformans habitats. Zbl Bakt Hyg I Abt Orig B 174, 179-186.

Torres-Rodríguez JM. 1993. Micosis sistémicas oportunistas excepcionales producidas por levaduras y hongos miceliares hialinos (hialohifomicosis). En: Torres-Rodríguez JM, del Palacio-Hernanz A, Guarro-Artigas J, Negroni Briz R, Pereiro-Miguens M (eds). Micología Médica. Masson, SA, Barcelona, España, Pp $205-$ 208

Tsai S, J Park, K Hirai, C Itakura. 1992. Aspergillosis and candidiasis in psittacine and passeriforme birds with particular reference to nasal lesions. Avian Dis 21, 699-709.

Velasco MC. 2000. Candidiasis and Cryptococcosis in birds. Sem Avian Exotic Pet Med 9, 75-81. 